\title{
Supply Chain Orchestration and Choreography: Programmable Logistics Using Semantics
}

\author{
S. Dalmolen ${ }^{1,2}$ H. M. Moonen ${ }^{1,2}$ J. van Hillegersberg ${ }^{1}$ \\ ${ }^{1}$ Industrial Engineering \& Business Information \\ Systems \\ University of Twente \\ Enschede, The Netherlands \\ s.dalmolen@utwente.nl \\ hans.moonen@utwente.nl \\ j.vanhillegersberg@utwente.nl
}

\author{
A. J. R. Stoter ${ }^{2}$, E. Cornelisse ${ }^{2}$ \\ ${ }^{2}$ Business Consulting \\ CGI Netherlands \\ Rotterdam, The Netherlands \\ arjan.stoter@cgi.com \\ erik.cornelisse@,cgi.com
}

\begin{abstract}
Interoperability between enterprise systems in supply chains is increasingly essential for successful supply chain integration and key in the creation ofinnovative businesses / business models. In this paper we present a novel approach for supply chain choreography to support supply chain organizations in practice in creating seamless chain integration.
\end{abstract}

The aim we set ourselves in this paper is to proposean architecture to enable and/or improve the exchange of information between supply chain organizations. To address this challenge we followed the design science methodology. First, we derived to a body of knowledge combining literature - on supply chain collaboration and challenges, but also corresponding areas such as web services - with empirical knowledge gained from applied research and industrial projects we were and are involved in.

Secondly, we propose a semantic architecture that is able to support seamlessly integration and create an ecosystem where programmable logistics are the future. First implementations are promising, but also revealed a series of improvements to our architecture. Further research is required for more practical validation and improving the theory, and validation of the current research.

Keywords - business process management; programmable logistics; supply chain management; ontology; interoperability; choreography; enterprise systems

\section{INTRODUCTION}

To executesupply chain collaboration in an agile and responsive manner, there is a need for a business ecosystem that has common protocols and interfaces.Bharadwaj et al. [1]state: "During the last decade, the business infrastructure has become digital with increased interconnections among products, processes, and services. Across many firms spanning different industries and sectors, digital technologies (viewed as combinations of information, computing, communication, and connectivity technologies) are fundamentally transforming business strategies, business processes, firm capabilities, products and services, and key interfirm relationships in extended business networks."[1]. Findings from several ongoinginnovation programs ata European andnational level e.g. iCargo ${ }^{1}, \mathrm{CO}^{2}, 4 \mathrm{C}_{4} \mathrm{More}^{3}$ strongly confirm this belief. Moreover,this is reinforcedby semi-structured interviews we conducted with practitioners and supply chain organizations during the execution of the iCargo and 4C4More project.

To enable inter-firm relationships, Interoperability is of key importance. While usually not a Top-3 concern of organizations, it has been an important theme in both research and practice for a long time[2][3][4]. Nevertheless, not all challenges around supply chain interoperability have been addressed[5], one example being a lack of collaborative process analysis tools, as identified by Mallek et al. [6].Several standards are currently available within supply chains e.g. GS1, EDIFACT, CEN, however interoperability between these standards is challenging[4],[7].With the rise of the semantic web and various ontologies to improve the interoperability[8],there isan opportunity as well as a need for an ontology-based semantic interoperability framework to support enterprises in achieving adaptable, dynamic and flexible supply chain collaborations. We present a novel approach for supply chain choreography that support supply chain collaborationthrough creating seamless supply chain integration.

\section{RESEARCH MOTIVATION: ORCHESTRATION AND CHOREOGRAPHY}

Supply chain choreography is the orchestration of a set of organizations that are willing to collaborate whereby it is not mandatory that one party hasoverall control. The supply chainecosystem has common goals e.g. reduce carbon emissions or improve customer satisfaction, besides the individual organizational goals. All the organization's executable business processes require to be designed as a service, named a business service description (BSD). The business processes from individual organizations can then be

\footnotetext{
$1_{\text {http://www.i-cargo.eu/ }}$

2 http://www.co3-project.eu/

3 http://www.dinalog.nl/en/projects/r_d_projects/4c4more/
} 
orchestrated by linking BSDs together. A short, practical example: a supplier publishesits service(business process) of goods that need to be shipped to a retailer. A logistic service provider offers the service of bringing goods from A to B under certain conditions, to particular costs and with corresponding carbon emissions. By linking these business services together the ecosystem is able to orchestrate these business processes. However choreography goes one step further, Hofman et al.describe a series of business transactions [9]. Besides connecting the business processes choreography tracks all the information, data and messages between all the used and available services (offered business processes to the ecosystem). It is responsible for tracking the sequence of multiple services instead of orchestration by- and control of one party. Choreography gives organizations the ability to collaborate with one or more organization(s) in the ecosystem and the freedom to set-up several ways of working via a publish/subscribe mechanism. Fig. 1 gives an example of a supply chain choreography. Onehexagon represents a business service description (one specific executable business process from an organization). An order has some specific preconditions for shipping. The choreography gives -in this case- three possible "routes" to ship the order. Depending on the given preconditions (costs, $\mathrm{CO} 2$ or speed) the green, blue or red route is chosen.

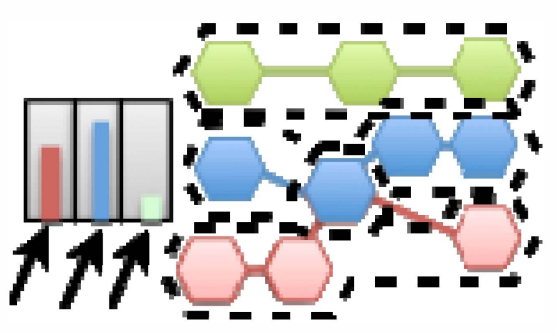

Fig. 1. Example of business choreography

Our starting point to improve supply chain collaboration in becoming more agile and flexibleis the supply chain information flow to improve optimization inreducingempty running and the use of scarce resources. We suggest the use of a network of ontologies[8] to support choreography. Using a network of ontologies allowing the IT infrastructures of supply chain organizations to adapt to the constant changing business environment.Our inspirationcame from web-service choreography which has been investigated for several years within web service development [10] and the first supply chain concepts of orchestration were proposedas "collaboration as a service"[11].In spite of these theories and proof of concepts,large scale supply chain service choreography in practice is rare.

Current IT systems in the supply chain are unable to support interoperability in an adaptable and flexible manner[12], [13]. In addition, existing legacy systems often lack functionalitythat is required for true dynamic end-to-end planning and choreography[13]. ERP systems in particular are commonly not suitable for sharing data or information among partners in the supply chain via a flexible manner. Easy interfaces or connections via standard APIs are still an issue.Integration costs are high and implementation timesare too long. Therefore the need arises for a flexible way of connecting systems to realize connections in a matter of days instead of development projects of months.

The main research question we address in this paper is:

[RQ] How can aneffective semantic architecturebe designed toenable and/or improve the exchange of information between supply chain organizations?

This research question isdivided into two sub questions:

[SQ1] What functionality is necessary for dynamically constructing and monitoring chains in logistic networks ata business level?

[SQ2] Which mechanism can effectively provide a semantic architecture forenabling choreography in logistic networks at the business level?

\section{METHOD}

Our research was built on existing literature of web service choreography and supply chain collaboration \& standards. We adopted the design science method [14], [15]. The research is explorative and practical whereby prototyping is essential. Therefore design science guidelines were followed described in[14].An iterative design cycle gives research the opportunity to learn quickly and contribute to the existing knowledge bases. Furthermore by following aniterative design cycle we can develop and validate different building blocks. The designed artifact "semantic architecture" needed to be applied in its intended environment as described by Hevner et al. [14].The research was carried out inthe Fast Moving Consumer Good supply chain. This industry sector is exemplary in facing social, regulatory and business changes that drive a need for supply chain collaboration and interoperability.

\section{SUPPLY CHAIN STANDARDS AND PRACTICAL USE}

Whipple, Lynch, and Nyaga[16]define supply chain collaboration asa long-term relationship where participants generally cooperate, share information, and work together to plan and even modify their business practices to improve joint performance[16].Supply chain collaboration requires standards for information exchange. Several standards are currently being used to achieve this goal, such as the common framework (CF) [17], the electronic data interchange for administration, commerce and transport (EDIFACT), extensible business reporting language (XBRL), the SAFE framework by the world customs organization (WCO), and arange of GS1 standards.

In practice, use of the above standards has several issues. Besides being numerous, they are commonly used for mass order planning. Exceptions, such as last minute orders or plan changes during transport often require manual interaction via phone or email. Second, coupling between systems (e.g. ERPs) is usually static and one-on-one. This leaves room for improvement in terms of efficiency and true dynamic end-toend planning and traceability.

\section{EVENT DRIVEN ARCHITECTURE\& ONTOLOGY BASED SYSTEMS}

To support supply chain choreography we decided to adopt an event-driven architecture (EDA) that is based on design 
patterns where events trigger actions to either process the incoming event and/or generate new events, building on top of the state of practice in service architecture - see for exampleHillegersberg et al. [18]. Using ontologies to describe events and rules decouples the functional meaning from a technical implementation. This has the advantage that people with software engineering skills can focus on realizing the technical implementation while others with better understanding of the business processes can focus on the functional aspects. Wang et al. [19] state that an ontology captures knowledge in related fields, provides a common understanding of domain knowledge, identifies common recognition vocabularies, and gives a clear definition of the relationship between these vocabularies from different levels of formal patterns. Using one ontology for describing both events and rules will make it easier to maintain consistency within the system.

\section{A. Semantic model}

The purpose of a semantic model (an ontology) is to assist software systems with the interpretation of the exchanged information between organizations and to invoke actions of these software systems. The relevant entities in a business process are called business objects, which can be either a thing, a person, an organization or even an abstract concept like a transport order.

A semantic model describes concepts and doesn't contain information about individual business objects. Dalmolen et al. [20] use the following definition of a semantic model: "is a collection of conceptual descriptions of business objects and their interactions that enable parties to interpret the meaning (semantics) and to act on the exchanged information".

For example, a truck will be described in a semantic model in terms of its attributes like a license plate for identification and a position to specify its movements. Also, the description of the format to identify a truck and how a position is expressed as a combination of time and location can be part of a semantic model. License plates numbers or positions of trucks itself are not stored in a semantic model but in datastores. Semantic models can be used to describe information about [20]:

- Business services from a provider or demand point of view to enable stakeholders of the supply chain to discover and find the appropriated business service automatically;

- Business objects and their interactions being subject to the business services; $\square$

- Business communities to define a common approach for collaboration by sharingthe same terminology, protocols and goals;

- Business messages, their meaning and role in the business process as well as a syntactical description of the message format;

- Business logic of a software service including the authorization and security protocols. $\square$
Currently, these descriptions are spread among multiple systems along the supply chain and mostly implemented as different incompatible information structures. Using a common technical approach to describe the different types of concepts as mentioned above, will enable a more consistent use of information.

Scheuermann and Leukel[21] performed a study that assesses the adoption of ontology engineering techniques in 16 Supply Chain ontologies. Next to the interesting overview their work provided, they articulated two main conclusions. The first, being a need for empirical research that evaluates ontology engineering artifacts in practice. Secondly, they made the observation that the field should learn from the conceptual modeling literature.

\section{ARTIFACT: SEMANTIC ARCHITECTURE}

Semantic architecture is thus a relatively novel topic in software architecture. In the context of this paper a semantic architecture describes the tools, concepts and process for seamless integration in a heterogeneous cross-domain environment. The goal of the semantic architecture is to provide interoperability in a flexible and responsive manner to enable and/or support supply chain choreography.

The artifact consists of four building blocks, namely supply chain data integration on a functional level; data security \& authorization, access points; choreography supporting mechanism

\section{A. Supply chain data integration on a functional level}

Current supply chain interoperabilityfaces two main challenges. Firstly, the world is changing in a rapid pace, so business models will change fast as well. Secondly, the supply chain environment itself can be characterized as being complex due to multiple organizations (cross-domain) and the heterogeneous aspect ofdifferent types and formats of data, and the timeliness of data.

Fig. 2 gives an overview of the challenges as described above from a business- and technical viewpoint. The vertical axis describes the business (driven) vision - follow arrow one. Modifications to business models require adequate data models to allow rapid adaption to change. The horizontal axis (arrow two) describes the technical vision to realize the business vision, and includes challenges in terms of security and compatibility with legacy systems. This study describes a first step to support adaptability and agility from both viewpoints. To create processes where companies can share data dynamically, adaptively, and secure, both the business (driven) vision and technical vision should be taken into account. To reduce complexity we propose three categories of handling data, described below and shown inFig. 2.

Interpretation of data: to facilitate the interpretation of information based on message standards e.g. as described in a Common Framework [CF]. The objective is to increase semantic interoperability among organizations with heterogeneous information systems in order to facilitate the communication, understanding and exchange of resources and information. 
Accessibility of data: to enable controlled and secure access without the need to know how and where the data is stored and which type of hardware, operating system and database are being used. The information can be accessed via a single access point even if the data is being stored in multiple databases. The objective is to provide easy and seamless connectivity.

Organization of data: an entity-centric approach[20] is used to reduce the complexity of the supply chain into individual and smaller parts. The information about each entity is being collected, stored, processed and shared by one software component that acts and serves in the best interest of that entity.

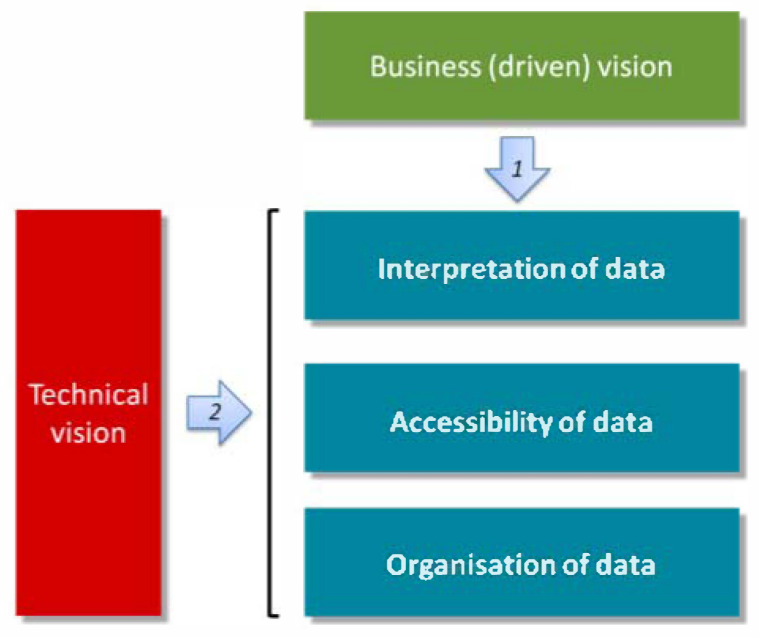

Fig. 2. Supply chain data integration

\section{B. Data security\& authorization}

A practical barrier today is the diversity of systems, devices and protocols being used in logistics supply chains. Virtualization of information provides the means for a technical infrastructure that abstracts the user from all the technical complexities of interoperability for collaboration and open exchange of information within communities. It allows easy and seamless communication by increasing the accessibility of data but with strict authorization control. It enables the dynamic configuration of communities to exchange information efficiently and effectively.

The ability to access information more easily does notmean that all information will be available toall. Business requirements still need a solid authorization mechanism to protect information from the competition or against criminal intentions. A direct consequence is the need for proper authentication before access can be granted or denied.

Even if the information is publically available, authentication is still required to reduce misbehavior and malicious activities. Therefore "public" information is not classified as "unrestricted" because access will be refused if the identity of the user or service cannot be verified. Fig. 3shows the expected security levels according to ISO/IEC 15408 standard.
We adoptthree levels of privacy:

- Public, free for the world but only supported via a trusted public service;

- $\quad$ Restricted, accessible for registered users (businessto-business);

- Confidential, only accessible for the information owner, which is the default configuration.
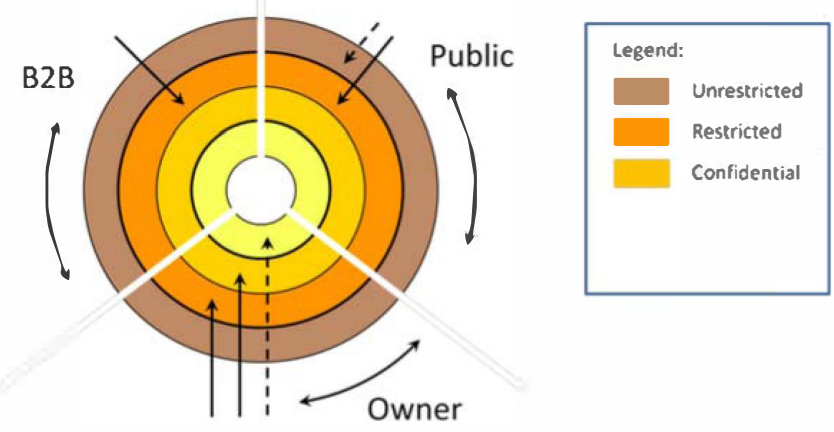

Fig. 3. Security levels

Information with the privacy classification "public" will be accessible via an API. Information that is publically accessible is disclosed as separate and dedicated services to ensure the security, responsiveness and availability of the restricted and confidential information.

Information is confidential by default and will only be shared after explicit authorization of the information owner. The authorization rights are part of the semantic model to be used within a business community. This design decision will enable a consistent and uniform security mechanism among different types of business objects. Consistency will increase the user comfort and a uniform mechanism will reduce the amount of software bugs.

The authorization rules are stored in the knowledge base of an application or software service. A tight relation with the defined concepts in a semantic model enables tooling and humans to address these rules at a functional level instead of a specialized database administrator level.

\section{Access points}

The third building block we define is an Access point. An access point is the main entry point to the ecosystem and provides controlled access to organizations business (software) services and can be seen as the "glue" in the ecosystem. Each organization requires an access point to join the ecosystem. Inside an access point, all information should belong to the same owner; see Fig. 3 about the security levels. A business service description is the virtual representation of a logistic business service and stored and accessed via Access Points. An organization connects its business services to their own access points. Within that specific access point the organization can configure the authorization of their business services and define which other organizations are allowed to use published business services.

Access pointscan collaborate (see Fig.4) to enable exchange of information between access points about logistic services, 
semantic models, logistic status information and the capabilities of software services. To discover other access points, access points can exchange information about themselves and information about business (software) services.Access points have a discover functionality to avoid the need for a central repository or central control in general.

\section{Choreography supporting mechanism}

Connecting the first three building blocks together will create a choreography supporting mechanism as shown in Fig.4. It shows three layers, the lowest layers is the infrastructure layer to support choreography and making logistics "programmable". At the infrastructure layer the semantic models are saved in the semantic repository.A business model analyst will use existing standards and tailor made extensions to describe all applicable business objects in the terminology of an organization. Then the new semantic model is stored in and available from a Semantic Repository.To organize the data, we adopted an entity-centric approach, described in paragraph A. Each entity needs to be registered and saved in a repository, because an entity is part of a semantic model. A new business service description will be published in an Entity Registry and become available via an access point to become available to all members of the ecosystem and software services for choreography in particular. An entity registry is like the Yellow pages to enable lookup capabilities. The authorization mechanism is also provided at the infrastructure layer. It does not decide who is allowed to see the data.That functionality is proprietary to an access point.

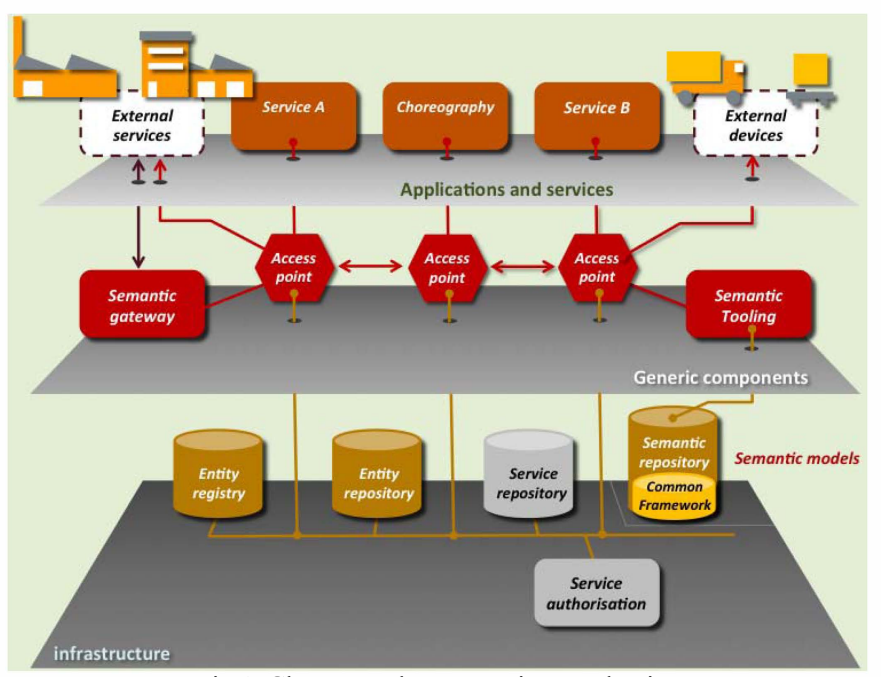

Fig.4. Choreography supporting mechanism

The middle layer is the actual transportation layer. Access points are connected. With a semantic gateway we are able to use current legacy systems. The semantic gateway gives connectivity to external services. Configuring an access point and adopting one or more semantic models and/or using existing logistic standards require tooling. Therefore we include semantic tooling that is able to integrate/connect semantic models/standards so seamless integration is available. The upper layer is the layer where organizations define their business services. Here we also include the choreography service that is using the access points and other business services to execute a choreography as shown in Fig. 1.

Once all relevant business objects for an organization are defined the user can start with the composition of complete door-to-door logistic servicesto create a possible route (see Fig. 1). In fact, programmable logistics can be composed by one or more users or be done by an algorithm depending of the defined business services. However in current practicesa user will usually develop a composition.

After the logistic chain is composed, the door-to-door service can be booked and executed. During the execution of the doorto-door logistic service, actual data is continuously collected by the different entities involved in the logistic chain and it can generate alerts triggered by specific events. This enables connected partners to optimize resources and to take counter measures in case of unexpected deviations such as delay. Also environmental parameters can be monitored in order to update service descriptions with actual environmental performances.

The following two levels of integration need to be addressed:

Message integration: two systems in order to be integrated do not need only to be connected but also to "speak the same language" at a functional level. The semantic architecture provides tools for describing the content of the published information in a way the other partners can understand, despite theirown specific structure.

System integration: to support the integration of existing software into the semantic architecture (Fig.4) at a technical level. For instance the ability to collect information stored in a database and publish that in a controlled way to the ecosystem via an Access Points.

The developed architecture is an open architecture. Other researchers and practitioners can adopt and develop access points or offer business/software services to the ecosystem. The architecture is based on distributed design and adopts the functionality of the Universal Description, Discovery \& Integration (UDDI) that is used for web services. UDDI is not heavily adopted in practice. However, as we make use of access points we thrive for an approach that is easier to adopt by practitioners.

\section{CONCLUSIONS AND DISCUSSION}

Existing Enterprise Systems omnipresent in supply chains face challenges to cope with the increasing need for flexibility and agility in information exchange between parties within the supply chain. Supply chains develop towardssupply networks, with trade relationships becoming shorter and more fluid[22], enabling new business models.Main challenge of these systems is the large amount of system integration needed to enable supply chain collaboration, by connecting information spread among multiple systems along the supply chain, systems that tend to have different incompatible information structures. Interoperability between systems is increasingly essential for successful supply chain integration.

This paperpresents an approach to provide flexibility and agility at the business level by using semantic models and an 
entity-centric approach.An open ecosystem is proposed, where organizations can come and go in offering their services (added value). Constructing new business models within supply chains require exchange of data cross-domain. This requires the functionality for seamlessly data integration, interpretation and organization (as was shown in Fig. 2).

Using the functionality of access points we support the organization of data and give a solution for the accessibility of data. Multiple standards are handled using semantic models. Additionally, we give organizations the ability to control their own data, and give other organizations the permission to subscribe to external data feeds. An event that occurs can be pushed towards the subscribed organizations.

In designing the proposed architecture for the exchange of information between supply chain organizations we made several design decisions based on prior literature, project experience and expert interviews.

To support choreography on business level we propose an architecture that uses semantic models and ontologies, in short:

- Define business service descriptions on a business level

- Business processes are mapped into semantic models, allowing flexible responses.

- Publish business services description.

- Configure access points by making explicit what services are subscribed, who has permission to use the service, and what specific organizations have access.

- Access Points discovery (providing connectivity).

Combining and implementing these components will result in an architecture that is fully operational and capable to compose door-to-door logistics supply chain solutions. When organizations change their business model the business service description needs to be changed and is able to integrate with other services.

Setting-up the business service descriptions was a particular challenge, as practitionersare clearly not used to think in services and ontologies. We continue our work in this area with more design iterations - focused on both the functionality, quality and usability of the architecture - to be tested in practice. The first design validations are currently being executed within the iCargo project via a pilot study.

We foresee more future research in validating and evaluating the proposed architecture in practice. From our perspective we have two main drivers. First, validating the flexibility of the current architecture at business level. Secondly, evaluate the semantic integration from different cross-domain data sources within a broader scope.

\section{ACKNOWLEDGMENT}

The research presented in this paper took and takes place within the "Intelligent Cargo in Efficient and Sustainable Global Logistics Operations" aka iCargo project (t\# 288383), which is partially funded by the European Commission as part of the FP7 program. This research takes place within the
4C4More project funded by Dinalog, the Dutch Logistical Top Institute.

\section{REFERENCES}

[1] A. Bharadwaj, O. A. El Sawy, P. A. Pavlou, and N. Venkatraman, "Digital business strategy: toward a next generation of insights," MIS Q., vol. 37, no. 2, pp. 471-482, 2013.

[2] J. Luftman and B. Derksen, "Key issues for IT executives 2012: Doing More with Less," MIS Q. Exec., vol. 11, no. 4, pp. 207-218, 2012.

[3] J. Luftman and T. Ben-Zvi, "Key issues for IT executives 2009: Difficult economy's impact on IT," MIS Q. Exec., vol. 9, no. 1, pp. 203-213, 2010

[4] C. Steinfield, M. L. Markus, and R. T. Wigand, "Through a glass clearly: standards, architecture, and process transparency in global supply chains," J. Manag. Inf. Syst., vol. 28, no. 2, pp. 75-108, 2011.

[5] C. Steinfield, "Inter-Organizational Information Systems," in Computing Handbook Third Edition, vol. 2, CRC Press, 2014, pp. 691 .

[6] S. Mallek, N. Daclin, and V. Chapurlat, "The application of interoperability requirement specification and verification to collaborative processes in industry," Comput.Ind., vol. 63, no. 7, pp. 643-658, 2012.

[7] M. L. Markus, C. W. Steinfield, and R. T. Wigand, "Industry-wide information systems standardization as collective action: the case of the US residential mortgage industry," Mis Q., pp. 439-465, 2006.

[8] P. Haase, S. Rudolph, Y. Wang, S. Brockmans, R. Palma, J. Euzenat, and M. d' Aquin, "Networked ontology model," NeOnDeliv. D, vol. 1, 2006.

[9] W. Hofman, "Runtime Logistic Process Orchestration Based on Business Transaction Choreography," in Business Process Management Workshops, 2013, pp. 550-559.

[10] C. Peltz, "Web services orchestration and choreography," Computer, vol. 36 , no. 10 , pp. $46-52,2003$

[11] J. Hillegersberg, H. Moonen, and S. Dalmolen, "Coordination as a Service to Enable Agile Business Networks," in The Dynamics of Global Sourcing. Perspectives and Practices, vol. 130, J. Kotlarsky, I. Oshri, and L. Willcocks, Eds. Springer Berlin Heidelberg, 2012, pp. 164-174.

[12] G. J. Sharman, "E-Supply Chain Management: Venturing Beyond ECommerce," in 2001 Annual Conference Proceedings, 2002.

[13] T. H. Davenport and J. D. Brooks, "Enterprise systems and the supply chain," J. Enterp. Inf. Manag., vol. 17, 2004.

[14] A. R. Hevner, S. T. March, J. Park, and S. Ram, "Design science in information systems research," Mis $Q$., vol. 28, no. 1, pp. 75-105, 2004

[15] A. Hevner and S. Chatterjee, Design science research in information systems. Springer, 2010.

[16] J. M. Whipple, D. F. Lynch, and G. N. Nyaga, "A buyer's perspective on collaborative versus transactional relationships," Ind. Mark.Manag., vol. 39, no. 3, pp. 507-518, 2010.

[17] J. T. Pedersen, "One Common Framework for Information and Communication Systems in Transport and Logistics: Facilitating Interoperability," in Sustainable Transport, Springer, 2012, pp. 165196.

[18] J. Van Hillegersberg, R. Boeke, and W.-J. Van Den Heuvel, "Potential of Webservices to enable smart business networks," J. Inf. Technol., vol. 19, no. 4, pp. 281-287, 2004.

[19] J. Wang, W. Zuo, F. He, and Y. Wang, "A New Formal Description of Ontology Definition and Ontology Algebra," in Second International Symposium on Knowledge Acquisition and Modeling, 2009. KAM '09, 2009, vol. 1, pp. 363-366.

[20] Dalmolen, S, Moonen, H M, and Cornelisse, E, "Information architecture using a cargo centric approach - Digital shadows of real world objects," presented at the e-Freight Conference, 2012.

[21] A. Scheuermann and J. Leukel, "Supply chain management ontology from an ontology engineering perspective," Comput.Ind., vol. 65, no. 6, pp. 913-923, 2014.

[22] E. van Heck and P. Vervest, "Smart business networks: how the network wins," Commun. ACM, vol. 50, no. 6, pp. 28-37, 2007. 